\title{
Impact of the small-scale elongated filaments on the oceanic vertical pump
}

\author{
by Guillaume Lapeyre ${ }^{1}$ and Patrice Klein ${ }^{2}$
}

\begin{abstract}
Oceanic mesoscale eddies (with a diameter of 50-100 km) are known to be associated with significant vertical tracer fluxes in the upper few hundred meters. In particular, they are important for the biogeochemical system, accounting for 20-30\% of the vertical nutrient transport. However, estimates of the global tracer fluxes neglect the role played by thin elongated filaments (with a width of $5-10 \mathrm{~km}$ ). These sub-mesoscale structures are produced by eddy interactions and ubiquitous in regions between eddies. We use a Surface Quasi-Geostrophic model to quantify their impact on the net vertical tracer flux into the surface layers. We show that eddy interactions are an important source of tracer injection because they lead to the production of filaments and to large vertical velocities within these structures. This is attributed to the frontogenetic dynamics induced by the horizontal stirring processes. When taking into account this process for the global statistics of tracer injection, the tracer flux associated with the filaments is as significant as that associated with the eddies. This outcome points out the necessity to explicitly include the filamentation process in global ocean model studies.
\end{abstract}

\section{Introduction}

Mesoscale oceanic eddies (the ocean's weather systems) are ubiquitous features in infrared and color satellite images (Abraham et al., 2000). Such eddies involve significant Sea Surface Height (SSH), thermohaline and density anomalies. Their importance has been stimulated by recent studies that significantly strengthen the vision of an upper ocean crowded with a large number of strongly interacting eddies. Indeed numerical experiments in the North Atlantic basin have shown that horizontal resolution of $1.5 \mathrm{~km}$ leads to an explosion of eddies and to an eddy kinetic energy increase by a factor 10 compared with "eddy resolving" (with a $15 \mathrm{~km}$ resolution) simulations (Hurlburt and Hogan, 2000; Siegel et al., 2001). As a result, almost half of the total oceanic kinetic energy is contained within mesoscale motions. It is also recognized that oceanic eddies trigger a vertical pump (the so-called "eddy pumping" mechanism), that transport nutrients from deeper layers into surface layers and this process may strongly affect the oceanic primary production (McGillicuddy et al., 1998).

1. Laboratoire de Météorologie Dynamique, Paris, France.

2. Laboratoire de Physique des Océans, IFREMER, BP 70, 29280 Plouzané, France.email: pklein@ifremer.fr 
The dynamical properties of an oceanic turbulent eddy field have been theoretically and numerically elucidated (Rhines, 1979; McWilliams, 1984; Hua and Haidvogel, 1986; Larichev and Held, 1995; Smith and Vallis, 2001). Among noteworthy characteristics that emerge is the strong production of small horizontal scales and subsequent filaments that rapidly develop between the eddies (McWilliams, 1984; Hua et al., 1998; Lapeyre et al., 1999). These filaments are elongated and weakly energetic structures that are ejected from the eddies, reaching hundreds of kilometers in length and width smaller than $10 \mathrm{~km}$. They involve quite small SSH, thermohaline and density contrasts. These characteristics are consistent with very fine in situ observations (Ledwell et al., 1993; Rudnick, 2001) and $1 \mathrm{~km}$ resolution satellite (such as SEAWIFS or AVHRR) images. Some experimental and numerical studies have pointed out that filaments with large vorticity values can be associated with significant vertical velocity (Pollard and Regier, 1990; Rudnick, 1996; Spall, 1995; Legal et al., 2007) and that they may affect the spatial tracer distribution (Lévy et al., 2001; Lévy and Klein, 2004). However the impact, at a basin scale, of the vertical fluxes induced by these small-scale filaments is usually considered as negligible and therefore ignored by global ocean observations and models (McGillicuddy et al., 2003). On the contrary, we suggest here that small-scale filaments can have a significant global impact on the vertical fluxes of certain oceanic tracers, including important biogeochemical tracers.

The mechanism underplay is related to the frontogenesis dynamics (Hoskins et al., 1978) that affects the filaments and to the fact that these filaments cover a large area of the oceanic surface layer. Frontogenesis may be understood here as the vertical velocities resulting from the intensification of surface fronts by the effect of large-scale strain. Indeed these filaments involve (usually weak) density anomalies bounded by density fronts. These density anomalies are horizontally stirred by eddies and this filament elongation makes the density fronts to strengthen, and, at the same time their associated vertical shear to decrease, which causes a thermal wind imbalance(Hoskins et al., 1978; Klein et al., 1998). Appearance of this imbalance causes motions to depart from geostrophy and thus induces an ageostrophic circulation (and in particular vertical motions) to maintain the geostrophic balance. In QG approximation, the role of this ageostrophic circulation is to instantaneously restore the thermal wind balance, which leads to increase the vertical shear and to decrease the density gradients. The upward and downward vertical motions that results from this ageostrophic circulation inhibits the production of smallscale density fronts and readjusts the related horizontal jets. The consequence is that, for a narrow filament, the vertical velocity will tend to decrease (increase) the density anomaly inside the elongating (contracting) filaments (as sketched in Fig. 1). The wellknown Omega equation (Hoskins et al., 1978) expresses this dynamics in stating that the vertical velocity field is controlled by the straining of horizontal density gradients. This frontal dynamics usually occurs on a scale close to the width scale of the density fronts so that it may be vigorous when filaments are continuously produced by eddy interactions. 




Figure 1. Schematic illustration of the vertical velocity field developing as a response to the elongation of a warm filament embedded in a horizontal strain field.

Although these vertical motions have a weak effect on the net vertical flux of density, they have a quite different impact on the distribution of any tracer whose sources and sinks differ from those for density (Wirth et al., 1997; Klein et al., 1998). This is the case for nutrients, whose concentration is large in the deeper layers and almost zero in the upper illuminated layers because of the phytoplankton consumption. Since a tracer concentration cannot be negative, downward tracer fluxes from the upper layers should then be quite negligible compared to the upward fluxes. This creates an asymmetry for the nutrient flux, which implies that the filamentation process may induce a continuous and irreversible net upward transport of tracer through frontogenesis. This is not the case for density because density anomalies are usually smaller than the positive background value, and therefore, both positive and negative vertical velocities are relevant for the density evolution. Quantification of the tracer vertical transport in these filaments, relatively to that in the eddies, is the focus of the present study.

To explore this issue, we make use of numerical experiments of oceanic turbulence that retain the basic properties of the mesoscale and small-scale dynamics. It is based on the Surface Quasi-Geostrophic (SQG) model that is known to be pertinent to reproduce the three-dimensional dynamics of mesoscale eddies and submesoscale filaments in the upper oceanic layers (Lapeyre and Klein, 2006). In this model, the vertical velocity field can be computed analytically for all depths from the surface density field and obeys the classical Omega equation (Hoskins et al., 1978). This is a consequence of the close relation between horizontal and vertical scales that results from the uniform potential vorticity assumption. In comparison, to accurately determine these motions in primitive equation models, one would need both horizontal and vertical resolutions that explicitly resolve fine scales of fronts and filaments.

We will first describe succinctly the numerical model we use. Then we will highlight the general mechanism of eddy interactions that is responsible of the production of filaments and of strong vertical motions through frontogenesis dynamics. We will then examine the impact of the small-scale filaments in the tracer budget in a turbulent field of interacting eddies. 
We will only consider the case of eddy interactions (and not their interaction with a large-scale front) because these eddy interactions lead to more vigorous frontogenesis and subsequent vertical motions.

Before concluding, we will discuss first the strength of the vertical motions within filaments through a scaling analysis and then the importance of our results for the global nutrient flux in the ocean.

\section{Method}

The Surface Quasi-Geostrophic model (Held et al., 1995) is based on a small Rossby number approximation to the primitive equations and assumes the quasi-geostrophic potential vorticity to be uniform in the interior of the fluid, i.e.

$$
\nabla_{H}^{2} \psi+\partial_{z}\left(\frac{f^{2}}{N^{2}} \partial_{z} \psi\right)=0
$$

with,

$$
-\left.\frac{\rho_{0} f}{g} \partial_{z} \psi\right|_{z=0}=\rho_{s}
$$

$\psi$ is the streamfunction. $N$ and $f$, respectively the Brunt-Väisälä and Coriolis frequencies, are assumed to be constant. $\nabla_{H}$ is the horizontal gradient operator and $z$ the vertical coordinate. $g$ is the gravity constant and $\rho_{0}=1000 \mathrm{~kg} \mathrm{~m}^{-3}$. The flow is supposed to decay from the surface (i.e., $\psi \rightarrow 0$ as $z \rightarrow-\infty$ ). Then the dynamics is entirely described by the time evolution of the density at the surface, $\rho_{s}$, whose equation is

$$
\partial_{t} \rho_{s}+\boldsymbol{u}_{s} \cdot \nabla_{H} \rho_{s}=0
$$

$\boldsymbol{u}_{s}=\left(-\partial_{y} \psi_{s}, \partial_{x} \psi_{s}\right)$ is the surface horizontal velocity field with $\psi_{s}$ the surface streamfunction. $x$ and $y$ are the zonal and meridional coordinates and $t$ the time. Eqs. (1) to (3) is a close system that allows to retrieve the 3 -D circulation in the upper oceanic layers.

Solving (1) and (2) in the spectral Fourier space leads to

$$
\hat{\psi}(z)=\hat{\psi}_{s} \exp \left(\frac{N k z}{f}\right)
$$

where $\widehat{()}$ is the horizontal spectral transform and $k$ the horizontal wavenumber. Using the hydrostatic approximation (3), this gives

$$
\hat{\psi}(z)=\frac{g}{k \rho_{0} N} \hat{\rho}_{s} \exp \left(\frac{N k z}{f}\right)
$$


Then, within the QG framework, all dynamical variables can be expressed in terms of the surface density,

$$
\begin{aligned}
\hat{\rho} & =\hat{\rho}_{s} \exp \left(\frac{N k z}{f}\right), \\
u & =-\partial_{y} \psi, \\
v & =\partial_{x} \psi, \\
\hat{w} & =\frac{g}{N^{2} \rho_{0}}\left(-\nabla_{H} \cdot\left(\widehat{\boldsymbol{u}_{s} \rho_{s}}\right) \exp \left(\frac{N k z}{f}\right)+\nabla_{H} \cdot(\widehat{\boldsymbol{u} \rho})\right) .
\end{aligned}
$$

Thus one property of this balanced model is that not only the horizontal velocity field but also the vertical velocity can be easily retrieved at all depths using only the surface density field (Hakim et al., 2002; Lapeyre and Klein, 2006). This property again results from the uniform potential vorticity in the interior (1) that allows to relate the horizontal structure to the vertical one.

Lapeyre and Klein (2006) have shown that baroclinic unstable flows exhibit strong correlations between the ocean interior potential vorticity anomalies and the surface density anomalies. Using this property they have developped an "effective" SQG model that is essentially equivalent to that considered above and that captures the 3-D dynamics within the upper layers of the ocean. The pertinence of this model as a balanced-equation system has been validated with the Primitive Equation solution of the Antartic Circumpolar Current (that involves in particular a depth-dependent Brunt-Väisälä frequency). They have shown that such SQG model allows to reconstruct the 3-D dynamics of submeso and mesoscale structures within the first five hundreds meters from just the horizontal surface density field using an "effective" constant Brunt-Väisälä frequency. These results validate the use of an SQG model to simulate the submeso and mesoscale dynamics in the first five hundreds meters below the surface. LaCasce and Mahadevan (2006) have also demonstrated the relevance of this model for the upper oceanic layers using in situ data and comparing with SQG reconstruction using SST.

In addition to the SQG model, a generic tracer resembling nutrients has been considered. Its value is initially 0 in the upper 100 meters and 1 elsewhere and its time evolution is driven by the full 3-D velocity field. This situation applies to any tracer for which there is a permanent sink in the surface layers. This is the case for example for nutrients in oligotrophic regions of the ocean. Therefore the total tracer content later appearing within the upper 100 meters results only from vertical tracer fluxes associated with the vertical velocity at $100 \mathrm{~m}$. For the sake of simplicity, density is considered to be a function of temperature only (i.e. salinity is assumed constant). The numerical model we used is based on the numerics of the model of Hua and Haidvogel (1986) in a doubly-periodic domain with resolutions of $512 \times 512$ and $1024 \times 1024$. 


\section{Mechanisms of vertical tracer injection}

An important characteristics of the three-dimensional dynamics of filaments is the strength of the eddy interactions that sets up the vigour of the stirring processes and therefore the production of small horizontal scales (McWilliams, 1984; Lapeyre et al., 1999). This continuous straining will result in vertical motions by frontogenesis processes.

This role of eddy interactions can be illustrated by considering one extreme limit such as intermittent eddy merging (McWilliams, 1984; Kevlahan and Farge, 1997; Velasco Fuentes, 2001) that have been observed in the ocean (Creswell, 1982; Biggs and Muller-Karger, 1994). For that purpose we have performed an experiment involving just a dipole (formed by two close eddies of opposite sign) and its encounter with a third-much less energeticanticyclonic eddy (its temperature variance is ten times smaller than that of the dipole). This situation is similar to the one described by Kevlahan and Farge (1997) and can be thought of as a proxy for very strong interactions between eddies. Numerical results show that, when the dipole approaches the third eddy, the latter is transported quite passively by the flow driven by the former. Then the weakly energetic eddy is intensively and entirely stretched by the dipole into small-scale structures and ultimately merges with its same sign partner. At the end, a new asymmetric eddy dipole continues its route (Fig. 2a). During the different phases of the merging, the amplitudes of the temperature anomalies are almost unchanged within the dipole but there is a very strong production of small-scale filaments and fronts with weak temperature anomalies due to the intense stretching of the weaker eddy. As a result, a strong vertical velocity field quickly develops (Fig. $3 \mathrm{~b}$ between day 50 and 90). Tracer is injected quite efficiently at small scales in the surface layers and spreads out into filaments that surround the two energetic eddies or is trapped within the newly formed eddy (Fig. 2b). Compared with a situation where the dipole is alone (i.e. the weak eddy is absent), the eddy encounter and merging leads to an increase of $100 \%$ of the total
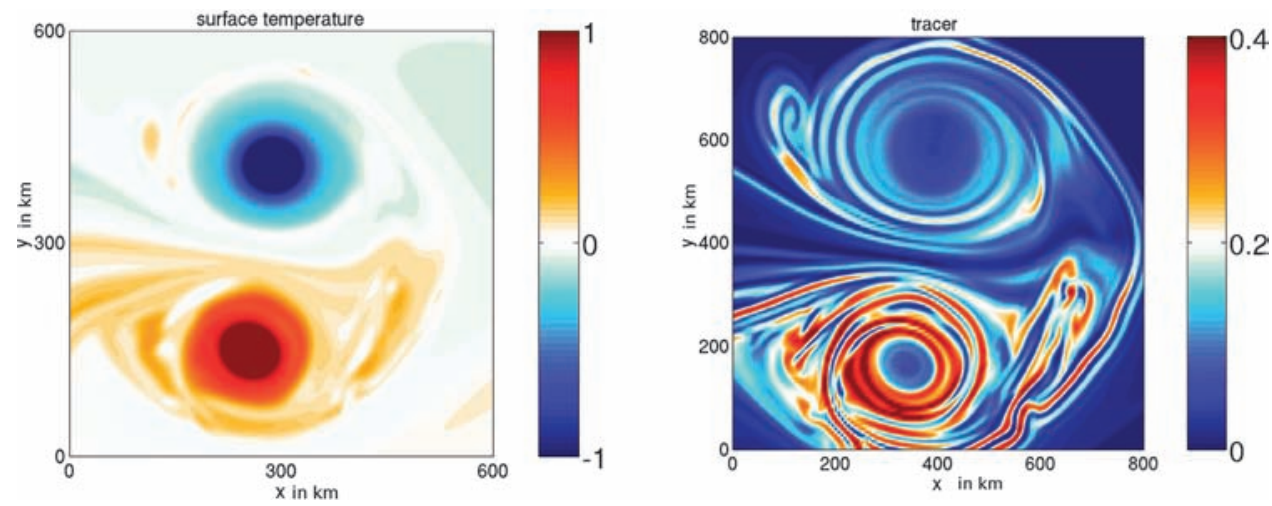

Figure 2. (a) Surface temperature anomaly (in Celsius degree), (b) tracer concentration in the upper $100 \mathrm{~m}$ from a simulation of three eddies after the merger of two of them. The newly formed eddy is at $x=250, y=150$. 

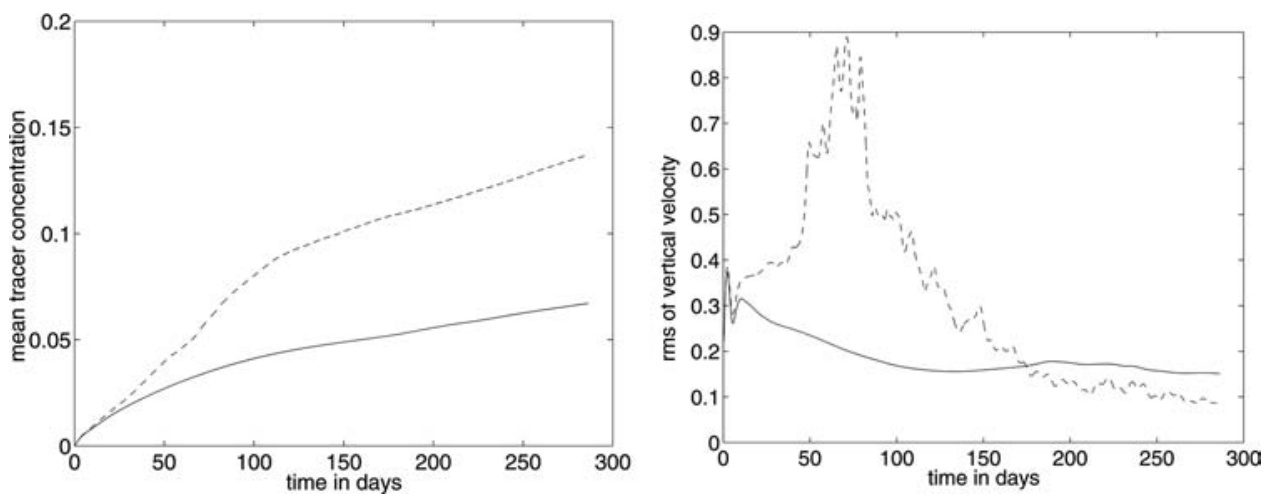

Figure 3. Time evolution (in days) of (a) mean tracer concentration, and (b) r.m.s. of vertical velocity at $z=-100 \mathrm{~m}$. The continuous curve corresponds to the evolution of a dipole without encounter, while the dashed line is the case of the encounter and the merger with a weak vortex (i.e. corresponding to Fig. 2).

tracer injection after 120 days (see Fig. 3a). This example well illustrates the crucial role of the eddy interactions that significantly pump tracer at small scales from below.

In a field involving a large number of interacting eddies, there will be a sustained production of horizontal small-scale filaments that can be either due to the intermittent eddy merging as in the preceding example or due to the continuous vortex stripping (Mariotti et al., 1994). The subsequent stirring of these structures by the strong deformation field induced by the eddies strengthens the submesoscale thermal fronts whose further evolution is driven by QG frontogenesis mechanisms(Hoskins et al., 1978). As mentioned in Section 1, the resulting vertical velocity field, although it limits or reduces the amplitude of small-scale temperature anomalies, can have a strong effect on the injection of any tracer absent in the surface layers. The preceding example of vortex interactions clearly emphasizes how the strong horizontal stirring of small-scale and weak temperature anomalies triggers a significant vertical pump. Then one may wonder whether such efficient and energetic dynamics has still a so significant impact on the vertical tracer injection in a fully turbulent eddy field.

\section{Turbulent eddy field experiment}

We now examine numerical simulations of a fully turbulent eddy field in free decay that represents the evolution of numerous eddies resulting from the active nonlinear interactions between them. Several experiments have been performed with this model, each one having a time duration of four months. Initial (random) conditions for the numerical experiments are similar to those in Hakim et al. (2002). Dimensional units are recovered using a length scale, $L=312 \mathrm{~km}$, a velocity scale, $U=2.2 \mathrm{~m} \mathrm{~s}^{-1}$, a Coriolis parameter, $f=7 \times 10^{-5} \mathrm{~s}^{-1}$, and a Brunt-Väisälä profile, $N^{2}=1.9 \times 10^{-5} \mathrm{~s}^{-2}$, leading to $U_{r m s}=0.19 \mathrm{~m} \mathrm{~s}^{-1}$ and 

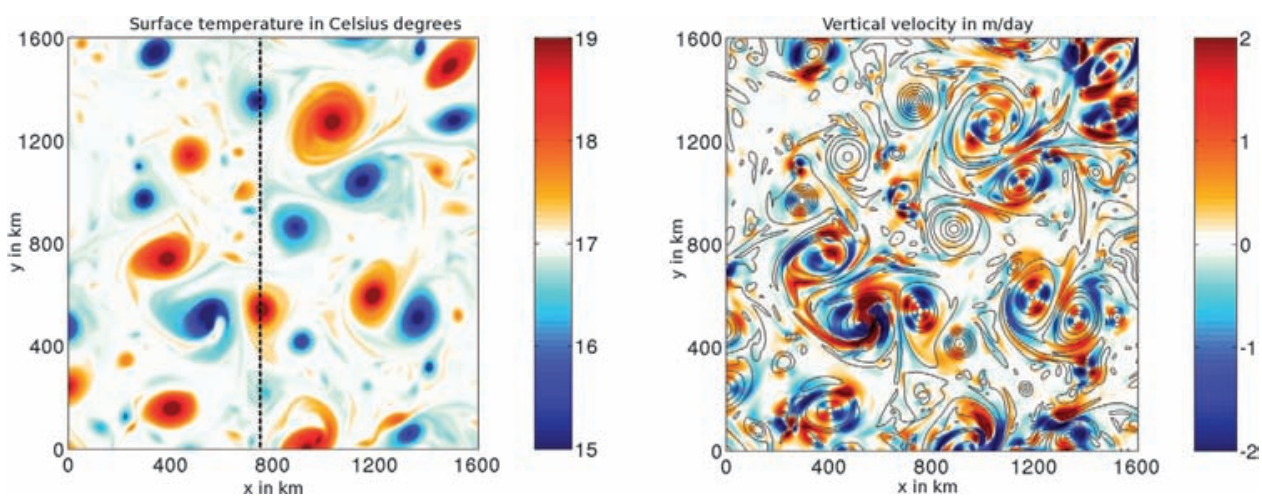

Figure 4. Horizontal snapshots of (a) surface temperature (in Celsius degree), and (b) vertical velocity at $z=-100 \mathrm{~m}$ (in meter per day). The figure corresponds to a simulation of a turbulent eddy field after 2 months of tracer injection. $x, y$ are in $\mathrm{km}$. Only a part of the domain is shown.

$\theta_{r m s}=0.47^{\circ} \mathrm{C}$. The best resolution used corresponds to a grid spacing of $\Delta x=\Delta y=$ $1.9 \mathrm{~km}$. Tracer units are kept nondimensional.

The surface temperature anomaly field, issued from the numerical experiments (Fig. 4a), displays classical patterns such as mesoscale eddies and small-scale-or submesoscalefeatures in between corresponding mostly to filaments. Cyclone (anticyclone) structures correspond to negative (positive) temperature anomalies. Computer animations reveal eddies strongly interacting with each other through mutual advection and deformation. Some are irreversibly deformed in long filaments by stronger partners while others merge, yielding both a single larger eddy and cast-off filaments ejected from the eddy cores. Vortices of opposite sign may also form dipoles that propagate rapidly until they eventually collide with a third vortex. These interactions between eddies are responsible of the continuous stretching and folding of small-scale filaments (Lapeyre et al., 1999; Lapeyre, 2002). The rapid evolution of both eddies and filaments is the signature of the inverse energy cascade (leading to larger eddies) and direct enstrophy cascade (continuous production of smaller and smaller scales leading to thinner and thinner filaments ultimately dissipated) that characterize the dynamics of such turbulent eddy field (McWilliams, 1984; Rhines, 1979).

To quantify the respective contributions of mesoscale $(50-100 \mathrm{~km})$ and submesoscale $(5-10 \mathrm{~km})$ structures to the vertical pump, the temperature anomaly field is used to partition the spatial field into eddies and filaments-"elongated" and "curved", each point of the grid belonging only to one category. Elongated filaments are mostly those located well outside the eddies whereas curved filaments are those that stay at their periphery. This is done in two steps. First a 2-D wavelet packet decomposition of the surface temperature field (Matlab package WaveLab) has been used to extract eddies. The decomposition uses an algorithm that finds a best basis for the Haar wavelets which selects the best location for the wave packets (Coifman and Wickerhauser, 1992). A thorough description of the method is given in Siegel and Weiss (1997). Then only 8\% of the wavelets with the largest amplitudes are 
Table 1. Partition between eddies and filaments of the total area $(S)$, temperature anomaly variance $\left(\theta^{2}\right)$, vertical velocity variance $\left(w^{2}\right)$ and total tracer amount in the first $100 \mathrm{~m}(C)$. Tables 1 and 2 have been averaged over 130 days.

$\begin{array}{lcccc}\text { Patterns } & \% S & \% \theta^{2} & \% w^{2} & \% C \\ \text { eddies } & 17 & 90 & 43 & 29 \\ \text { Curved filaments } & 26 & 4 & 22 & 24 \\ \text { Elongated filaments } & 57 & 6 & 35 & 47\end{array}$

retained and the signal is reconstructed. As these wavelets are localized in space, the resulting field partitions the space between eddies and filaments. These filaments are then separated into elongated and curved structures depending on the curvature of the temperature isocontours. This is done every two days. As this method is not Lagrangian, a point belonging to one category may belong to another category at a later time, such that there may be mass transfer between each category: typically when a filament is stripped out from a vortex, its material would first belong to the "eddy" category, then to the "curved filament" category, and if it is ejected far from the vortex, it would belong to the "elongated filament" category. Results are not very sensitive to the different thresholds used in these techniques, either for the eddy extraction or for the filament separation into the two classes. The resulting partition in terms of percentage of the total area and of the temperature variance between eddies and filaments (Table 1) is consistent with previous studies (Hakim et al., 2002) and confirms the efficiency of the extraction algorithms. This can be also assessed by comparing the temperature field of Figure $4 \mathrm{a}$ with the partition field of Figure 6. Number of eddies within the domain ranges between 40 and 50 depending on time, with their scales being from $100 \mathrm{~km}$ to $20 \mathrm{~km}$.

One important characteristics is that the eddies account for $90 \%$ of the temperature variance although they represent less than $20 \%$ of the total area (Table 1). As mentioned before, this result compares quite well with those from previous studies (Hakim et al., 2002). On the other hand, each class of filaments captures a very small part of the temperature variance: this is particularly true for the elongated filaments that capture only $6 \%$ of the temperature variance although they represent more than half of the total area (Table 1). In other words, large temperature anomalies are mostly found in mesoscale eddies whereas submesoscale elongated and curved filaments mostly concern weak temperature anomalies (Table 2).

Table 2. Detailed statistics of the r.m.s. (root mean square) values of the temperature anomalies $\theta_{\text {rms }}$ (in degrees Celsius), vertical velocity $w_{r m s}$ (in meter per day), and the mean tracer concentration in the first 100 meters $\bar{C}$.

Patterns 
We have calculated the vertical velocity related to the eddy field at a depth $z=-100 \mathrm{~m}$. One remarkable characteristic, robust at any time, is that $35 \%$ of the total vertical kinetic energy $\left(w^{2}\right)$ is located within the elongated filaments, i.e. well outside the eddies (Table 1). This result challenges the approach of previous studies on the tracer vertical injection that only focused on the eddies and their periphery. In physical space (Fig. 4b), the $w$-field involves small-scale hot spots with alternate signs organized as dipolar patterns in filaments and quadrupolar patterns in eddies. Such organization in quadrupolar and dipolar structures is consistent with results of primitive equation simulations (Lévy et al., 2001; Lapeyre and Klein, 2006). The significant kinetic energy of the vertical motions well outside the eddies is explained by the larger area occupied by the elongated filaments (Table 1) and also by the local values of the vertical velocity (whose magnitude can reach $10 \mathrm{~m}$ per day) that are only twice smaller than those in the eddies (as revealed by the $w_{r m s}$-values in Table 2). In comparison, the temperature anomalies are statistically six times smaller in the elongated filaments that within the vortices. The mechanisms that drive such vertical motions in regions outside the eddies are interpreted in terms of the filament stirring due to the eddy interactions and subsequent ageostrophic dynamics described in the previous sections.

These characteristics of the vertical velocity field raise the following question: will a vertically injected tracer (such as nutrients) be mostly found within mesoscale eddies or well outside the eddies? We have addressed this question by analyzing the tracer evolution within the first 100 meters (again, initial tracer field has a horizontally uniform background value of 0 in the $100 \mathrm{~m}$-deep surface layers and 1 below). The impact of the filaments on the tracer vertical transport, relatively to that of the eddies, is illustrated by the comparison of Figures $4 \mathrm{a}$ and $5 \mathrm{a}$ that shows the different spatial organization of tracer and temperature and the predominance of small-scale filament-like patterns in the tracer field. Temperature anomalies between the eddies are rather small while significant amounts of tracer are found not only within the eddies but, also well between the eddies. These characteristics are also confirmed by Figure $5 \mathrm{~b}$ that shows a typical vertical section through the turbulent eddy field: one sees large temperature anomalies related to eddies having almost circular structures with diameter as large as $100 \mathrm{~km}$. Between the eddies, a dense population of filaments with very weak temperature anomalies can be found. These filaments involve much smaller horizontal scales, as small as $10 \mathrm{~km}$ in one direction. Figure 5b, as Figure 5a, clearly shows that significant amounts of tracer within the upper $100 \mathrm{~m}$ (indicated by a small "nitracline" depth) occur within eddies. But these figures also reveal that quite non negligeable amounts of tracer occur between the eddies. Furthermore the tracer in the regions between eddies (Fig. 5b) appears to have a large spatial variability despite the weak small-scale temperature anomalies there. This illustrates clearly that the vertical velocity field has a weak effect on temperature field but a strong effect on the tracer field and this is due to the different vertical mean gradients for temperature and tracer. The mechanism sketched in Figure 1 explains why we can find large values of tracer in these filaments.

One may ask whether the tracer found between the eddies, in particular within the elongated filaments, is mostly injected within these structures or horizontally advected from 

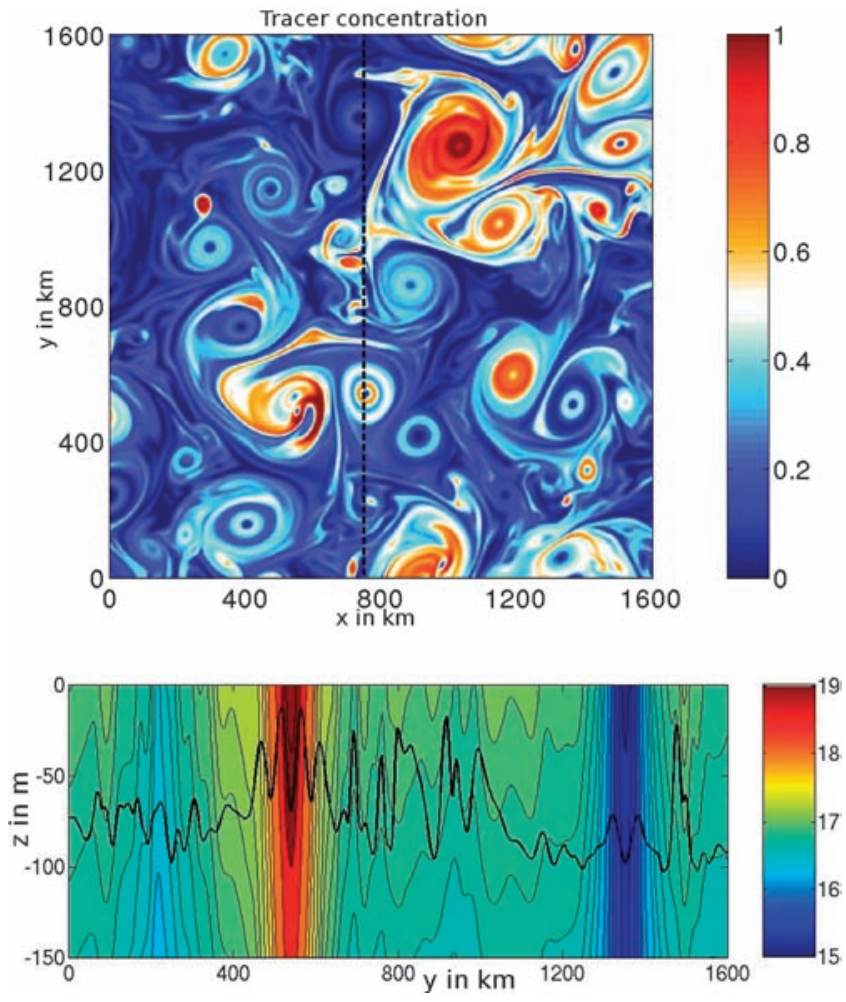

Figure 5. (a) Horizontal snapshot of the tracer concentration $C$ for the simulation of Figure 4. (b) vertical section of temperature (see color scale) and equivalent "nitracline" depth (black solid line) defined as $H(1-C)$, with $H=100 \mathrm{~m}$.

other regions (such as the eddies or curved filaments around the eddies). The answer is provided by Figure 7 that displays the net tracer supply in the upper layers: almost $50 \%$ of the vertical tracer fluxes occurs within the elongated filaments throughout the course of the experiments. On the other hand, almost $50 \%$ of the total tracer content is found at any time within the elongated filament region (Table 1). These results, about the vertical tracer fluxes and tracer content, and the similarity of the ratios $\bar{C} / w_{\text {rms }}$ (Table 2) found in the three regions explicitly point out that it is the vertical transport, and not the horizontal one, that contributes to the spatial organization of the tracer field. This clearly emphasizes the strong potential of filaments to act as a vertical pump which locally fluxes tracers upwards between the eddies.

\section{Discussion}

The robustness of the results displayed in Table 2 about the vertical motion strength within small-scale filaments with weak density anomalies can be verified through a scaling 


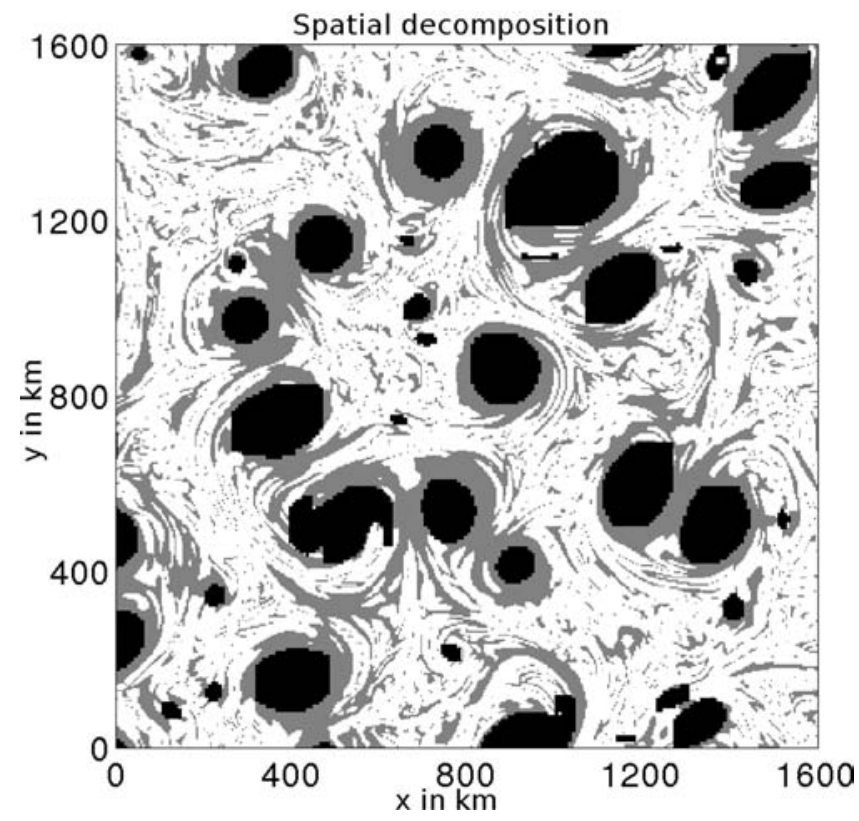

Figure 6. Decomposition of the mesoscale field in different dynamical structures using the wavelet method. In black, vortices, in gray the rounded filaments and in white the elongated filaments. The field corresponds to Figure 4.

analysis. As explained in Section 1, these filaments are bounded by density fronts. These fronts strengthen when the filament is elongated by a large-scale strain field, which leads to a thermal wind imbalance. The ageostrophic circulation, and in particular the vertical motions, act to restore the thermal wind balance. All these mechanisms are expressed in the well-known Omega equation (Hoskins et al., 1978) that reads (for a density front aligned in the $y$-direction):

$$
N^{2} w_{x x}+f^{2} w_{z z}=-2 \frac{g}{\rho_{0}} \partial_{x}\left(\partial_{x} u \partial_{x} \rho\right)
$$

In this equation, the RHS represents the forces that tend to destroy the thermal wind balance and the LHS those that restore this balance.

A simple expression for the vertical velocity, $W$, can be derived from (9) if we assume that the horizontal length scale $\left(L_{s}\right)$ of the strain field $\left(\partial_{x} u\right)$ is much larger than the width scale of the filaments $(L)$ and that $\partial_{x} u$ and $\rho$ decays exponentially with a vertical decay scale respectively equal to $D_{s}=f L_{s} / N$ for the strain and $D=f L / N$ for the density (as indicated in Section 2). Then, using $-W / L^{2}$ and $-\Delta \rho / L^{2}$ as scales for $\partial_{x x} w$ and $\partial_{x x} \rho$ and $\sigma$ a scale for the surface large-scale strain field $\left(\partial_{x} u\right)$, this expression is:

$$
W(z)=\frac{g \sigma}{N^{2}} \frac{\Delta \rho}{\rho_{0}} \frac{z}{D} \exp (z / D)
$$






Figure 7. Time evolution of the integrated tracer vertical flux $\left(\int_{0}^{t} \frac{w C}{H} d t\right)$ for the eddies, curved filaments (C Fil) and elongated filaments (E Fil), normalized by the area percentage (Table 1). $H$ is equal to $100 \mathrm{~m}, t$ is the time (in days) and $w$ the vertical velocity. $C$ is equal to 1 when $w>0$ and equal to the tracer concentration in the first $100 \mathrm{~m}$ when $w<0$. Abscissa and ordinate units are respectively days and tracer concentration units.

If we use classical values related to small-scale filaments, i.e. $N / f=62.5, L=20^{4} \mathrm{~m}$, $\sigma=2.5 \times 10^{-6} \mathrm{~s}^{-1}$, as well as $\Delta \rho \approx \alpha \rho_{0} \theta_{r m s}$ (with $\alpha=2 \times 10^{-4}$ the thermal expansion coefficient and $\theta_{r m s}$ the value given in Table 2 for the elongated filaments), we get $D=$ $320 \mathrm{~m}$ and $W(100 \mathrm{~m}) \approx 0.85 \mathrm{~m} \cdot \mathrm{d}^{-1}$. This value is close to the $w_{r m s}$-value indicated in Table 2 for the elongated filaments. Also $W$ reaches its maximum value near $D=320 \mathrm{~m}$. In regions of strong stratification (when $N$ is larger), the decay scale will be much smaller and the vertical velocity will be concentrated in the few upper tens of meters. This can prevent vertical transport of nutrients if the nitracline is deeper. Thus the frontogenesis processes well explain the magnitude of the vertical motions within the elongated filaments. Furthermore (10) displays the dependency of the vertical motions within filaments to the parameters of the system. Filaments of $6.25 \mathrm{~km}$ width have the maximum vertical motions at $100 \mathrm{~m}$. Wider or narrower filaments will have a smaller $100 \mathrm{~m}$ vertical velocity. At last, since the strain rate is related to the energy of the eddies, these vertical motions will increase with the kinetic energy of the eddies if we assume that the relative magnitude of the $\theta_{r m s}$ within filaments and eddies is conserved when this energy varies.

Relevance and importance of our results can be checked through the estimation of the global nutrient budget since nutrients are a class of tracers that has been intensively observed in the oceans for a long time. The existing large datasets, combined with rough estimations of the vertical mixing and vertical velocity field, have allowed to assess the nutrient budget within the upper oceanic layers. One outcome is that the eddy-driven vertical injection of 
nutrient from deeper layers into the upper layers represents 20 to $30 \%$ of the total nutrient annual transport (McGillicuddy et al., 2003). This assessment accounts for only the vertical injection within the eddies. From our results, we can estimate the contribution of each component (eddies and two classes of filaments) to the annual nutrient supply to the upper layers, and compared these estimates with those obtained from oceanic observations. Here nitrates is used as the nutrient, with units of mol $\mathrm{N}$ per $\mathrm{m}^{2}$. We take a value of $1.5 \mathrm{mmol}$ $\mathrm{N} \mathrm{m}^{-3}$ for the nitrate below $100 \mathrm{~m}$ and use, for upward velocities, the values of $w_{\text {rms }}$ (Table 1) normalized by half of the area percentage (Table 2). Then our estimates yield a contribution of respectively $0.08 \mathrm{~mol} \mathrm{~N} \mathrm{~m}^{-2} \mathrm{yr}^{-1}$ for the eddies, $0.06 \mathrm{~mol} \mathrm{~N} \mathrm{~m}^{-2} \mathrm{yr}^{-1}$ for the curved filaments and $0.12 \mathrm{~mol} \mathrm{~N} \mathrm{~m}^{-2} \mathrm{yr}^{-1}$ for the elongated filaments. When only the eddy and curved filament contributions are taken into account, our estimate $(0.14 \mathrm{~mol}$ $\mathrm{N} \mathrm{m}^{-2} \mathrm{yr}^{-1}$ ) compares well with previous estimates of the total eddy contribution (about $0.12 \mathrm{~mol} \mathrm{~N} \mathrm{~m}^{-2} \mathrm{yr}^{-1}$ (McGillicuddy et al., 1998, 2003)) which ignore the regions between the eddies. This good comparison with observations of the statistical impact of the eddies strengthens the importance of our main result: when the contribution of elongated filaments is taken into account, the total contribution to the nutrient supply attains $0.26 \mathrm{~mol} \mathrm{~N} \mathrm{~m}^{-2}$ $\mathrm{yr}^{-1}$ and almost doubles the previous global geochemical estimates.

\section{Conclusion}

In this study, we have examined the impact of eddy interactions and subsequent 3-D dynamics of filaments on the vertical injection of tracers. For this purpose, we have used simulations of a Surface Quasi-Geostrophic model that allows to capture the basic characteristics of the meso and submeso scales. One of our finding is that whereas the vertical velocity field inhibits the production of small scales of temperature anomalies outside the eddies, on the contrary it strongly stimulates the injection of tracers at small scales. This is only due to the tracer large-scale background field that differs from that of temperature.

Our results reveal that a significant part of the eddy-induced vertical injection of tracer (such as nutrients into the surface waters) takes place well outside the eddies in small-scale filament-like structures involving weak temperature anomalies. The consequence would be to almost double previous estimates of the total tracer injected, since this injection was assumed to principally occur within eddies (McGillicuddy et al., 1998) or thermal fronts with large temperature anomalies (Spall and Richards, 2000). The mechanisms involved in this injection, usually missed in most observational studies (because of the sampling strategy) and in most eddy resolving models, strongly highlight the need (also emphasized in other studies (Lévy et al., 2001; Mahadevan and Archer, 2000)) to use a much higher spatial resolution than previously considered.

Such an impact of the filaments on the vertical tracer fluxes is an important issue in the context of physical-biological interactions (Jenkins and Goldman, 1985) since nutrients can be efficiently pumped by eddies from deeper layers into the surface layers (McGillicuddy et al., 2003). Within this context the present analysis may provide an answer to the ongoing 
debate about which physical mechanisms can close the nutrient balance in the oceans (McGillicuddy et al., 1998; Oschlies and Garçon, 1998; McGillicuddy et al., 2003; Abraham et al., 2000). No biological model has been used in this study since we only focus on the physical processes that affect the vertical transport. In light of results of Martin et al. (2002), we might expect that this injection of nutrient at small scales in regions of vigorous horizontal stirring can increase dramatically the primary production. Also the mechanism we describe may provide an alternate explanation of the small-scale plankton patchiness observed in high resolution satellite images (Abraham et al., 2000; Mizobata et al., 2002): this patchiness can result not only from the horizontal stirring of larger scale patterns (Abraham, 1998; Dandonneau et al., 2003; Killworth et al., 2004), but also from significant local vertical transport of nutrients within small-scale structures.

Acknowledgments. This work is supported by the CNRS and IFREMER (FRANCE). Some of the calculations reported here were done on the NEC SX5 of the IDRIS (Orsay, France). We thank both reviewers for their helpful comments.

\section{REFERENCES}

Abraham, E. R. 1998. The generation of plankton patchiness by turbulent stirring. Nature, 391, 577-580.

Abraham, E. R., C. S. Law, P. W. Boyd, S. J. Lavender, M. T. Maldonado and A. R. Bowle. 2000. Importance of stirring in the development of an iron-fertilized phytoplankton bloom. Nature, 407, $\underline{727-730 .}$

Biggs, D. C. and F. E. Muller-Karger. 1994. Ship and satellite observations of chlorophyll stocks in interacting cyclone-anticyclone eddy pairs in the western gulf of mexico. J. Geophys. Res., 99, 7371-7384.

Coifman, R. and M. V. Wickerhauser. 1992. Entropy-based algorithms for best basis selection. I. E. E. E. Trans. Info. Theory, 38, 713-718.

Creswell, G. R. 1982. The coalescence of two east Australian Current warm core eddies. Science, $215,161-164$.

Dandonneau, Y., A. Vega, H. Loisel, Y. du Penhoat and C. Menkes. 2003. Oceanic rossby waves acting as a "hay rake" for ecosystem floating by-products. Science, 302, 1548-1551.

Hakim, G. J., C. Snyder and D. J. Muraki. 2002. A new surface model for cyclone-anticyclone asymmetry. J. Atmos. Sci., 59, 2405-2420.

Held, I. M., R. T. Pierrehumbert, S. T. Garner and K. L. Swanson. 1995. Surface quasi-geostrophic dynamics. J. Fluid Mech., 282, 1-20.

Hoskins, B. J., I. Draghici and H. C. Davies. 1978. A new look at the $\omega$-equation. Q. J. R. Meteorol. Soc., 104, 31-38.

Hua, B. L. and D. B. Haidvogel. 1986. Numerical simulations of the vertical structure of quasigeostrophic turbulence. J. Atmos. Sci., 43, 2923-2936.

Hua, B. L., J. C. McWilliams and P. Klein. 1998. Lagrangian accelerations in geostrophic turbulence. J. Fluid Mech., 366, 87-108.

Hurlburt, H. E. and P. J. Hogan. 2000 . Impact of $1 / 8^{\circ}$ to $1 / 64^{\circ}$ resolution on gulf stream model-data comparisons in basin-scale subtropical atlantic ocean models. Dyn. Atmos. Oceans, 32, 283-329.

Jenkins, W. J. and J. Goldman. 1985. Seasonal oxygen cycling and primary production in the Sargasso Sea. J. Mar. Res., 43, 465-491. 
Kevlahan, N. K.-R. and M. Farge. 1997. Vorticity filaments in two-dimensional turbulence: creation, stability and effect. J. Fluid Mech., 346, 49-76.

Killworth, P. D., P. Cipollini, B. M. Uz and J. R. Blundell. 2004. Physical and biological mechanisms for planetary waves observed in sea-surface chlorophyll. J. Geophys. Res., 109(C7), 10.1029/ 2003JC001768.

Klein, P., A.-M. Tréguier and B. L. Hua. 1998. Three-dimensional stirring of thermohaline fronts. J. Mar. Res., 56, 589-612.

LaCasce, J. H. and A. Mahadevan. 2006. Estimating sub-surface horizontal and vertical velocities from sea surface temperature. J. Mar. Res., 64, 695-721.

Lapeyre, G. 2002. Characterization of finite-time lyapunov exponents and vectors in two-dimensional turbulence. Chaos, 12, 688-698.

Lapeyre, G. and P. Klein. 2006. Dynamics of the upper oceanic layers in terms of surface quasigeostrophy theory. J. Phys. Oceanogr., 36, 165-176.

Lapeyre, G., P. Klein and B. L. Hua. 1999. Does the tracer gradient vector align with the strain eigenvectors in 2-D turbulence? Phys. Fluids, 11, 3729-3737.

Larichev, V. D. and I. M. Held. 1995. Eddy amplitudes and fluxes in a homogeneous model of fully developed baroclinic instability. J. Phys. Oceanogr., 25, 2285-2297.

Ledwell, J. R., A. J. Watson and C. S. Law. 1993. Evidence for slow mixing across the pycnocline from an open-ocean tracer-release experiment. Nature, 364, 701-703.

Legal, C., P. Klein, A.M. Treguier and J. Paillet. 2007. Diagnosis of the vertical motions in a mesoscale stirring region. J. Phys. Oceanogr., (in press).

Lévy, M. and P. Klein. 2004. Does the low frequency of the mesoscale dynamics explain a part of the phytoplankton and zooplankton spectral variability. Proc. Roy. Soc. London Ser. A, 460, 16731687.

Lévy, M., P. Klein and A.-M. Tréguier. 2001. Impact of sub-mesoscale physics on production and subduction of phytoplankton in an oligotrophic regime. J. Mar. Res., 59, 535-565.

Mahadevan, A. and D. Archer. 2000. Modeling the impact of fronts and mesoscale circulation on the nutrient supply and biochemistry of the upper ocean. J. Geophys. Res., 105, 1209-1225.

Mariotti, A., B. Legras and D. G. Dritschel. 1994. Vortex stripping and the erosion of coherent structures in two-dimensional flows. Phys. Fluids A., 6, 3954-3962.

Martin, A. P., K. J. Richards, A. Bracco and A. Provenzale. 2002. Patchy productivity in the open ocean. Glob. Geochem. Cycles, 16, 10.1029.

McGillicuddy, D. J., A. R. Robinson, D. A. Siegel, H. W. Jannasch, R. Johnson, T. D. Dickey, J. McNeil, A. F. Michaels and A. H. Knap. 1998. Influence of mesoscale eddies on new production in the Sargasso Sea. Nature, 394, 263-266.

McGillicuddy, D. J., L. A. Anderson, S. C. Doney and M. E. Maltrud. 2003. Eddy-driven sources and sinks of nutrients in the upper ocean: result from a $0.1^{\circ}$ resolution model of the north atlantic. Glob. Geochem. Cycles, 17, 1035.

McWilliams, J. C. 1984. The emergence of isolated coherent vortices in turbulent flow. J. Fluid Mech., $146,21-43$.

Mizobata, K., S. I. Saitoh, A. Shiomoto, T. Miyamura, N. Shiga, K. Imai, M. Toratani, Y. Kajiwara and K. Sasaoka. 2002. Bering sea cyclonic and anticyclonic eddies observed during summer 2000 and 2001. Prog. Oceanogr., 55, 65-75.

Oschlies, A. and V. Garçon. 1998. Eddy-induced enhancement of primary production in a model of the north atlantic ocean. Nature, 394, 266-269.

Pollard, R. T. and L. A. Regier. 1990. Large variations in potential vorticity at small spatial scales in the upper ocean. Nature, 348, 227-230.

Rhines, P. B. 1979. Geostrophic turbulence. Annu. Rev. Fluid Mech., 11, 404-441. 
Rudnick, D. L. 1996. Intensive surveys of the azores front. 2. inferring the geostrophic and vertical velocity fields. J. Geophys. Res., 101, 16,291-16,303.

2001. On the skewness of vorticity in the upper ocean. Geophys. Res. Lett., 28, 2045-2048.

Siegel, A. and J. B. Weiss. 1997. A wavelet-packet census algorithm for calculating vortex statistics. Phys. Fluids, 9, 1988-1999.

Siegel, A., J. B. Weiss, J. Toomre, J. C. McWilliams, P. S. Berloff and I. Yavneh. 2001. Eddies and vortices in ocean basin dynamics. Geophys. Res. Lett., 28, 3183-3186.

Smith, K. S. and G. K. Vallis. 2001. The scales and equilibration of midocean eddies: freely evolving flow. J. Phys. Oceanogr., 31, 554-571.

Spall, M. A. 1995. Frontogenesis, subduction, and cross-front exchange at upper ocean fronts. J. Geophys. Res., 100, 2543-2557.

Spall, S. A. and K. J. Richards. 2000. A numerical model of mesoscale frontal instabilities and plankton dynamics: I model formulation and initial experiments. Deep-Sea Res., 47, 1261-1301.

Velasco Fuentes, O. U. 2001. Chaotic advection by two interacting finite-area vortices. Phys. Fluids, 13, 901-912.

Wirth, V., C. Appenzeller and M. Juckes. 1997. Signatures of induced vertical air motion accompanying quasi-horizontal roll-up of stratospheric intrusions. Mon. Weather Rev., 125, 2504-2519.

Received: 18 December, 2005; revised: 26 September, 2006. 